\title{
The Constitution of New Media Art in Space
}

\author{
Yuqing Lei ${ }^{1, *}$ \\ ${ }^{1}$ Shanghai Academy of Fine Arts, Shanghai University, Shanghai 200040, China \\ *Corresponding author. Email: greyleeee@qq.com
}

\begin{abstract}
As a digital technology or a more extensive information medium, new media has begun to enter the lives of the masses, and participates in space construction in the form of works or advertisements, affecting people's lifestyle and the value of space. This is a new way of information transmission, and also a mapping of future space appearance and development. Since the development of the city, designers have been constantly exploring innovative ways and experience ways in the process of space establishment, and they have turned their attention to the design of information transmission. In this paper, space is divided into three categories, showing cases of new media art in exhibition space, public space and urban space respectively, and through the analysis of these cases, the ways and possibilities of new media art acting on space design in the context of the development of new media technology are discussed. Through the study of this paper, it can be found that new media art, as a design method, is stationed in the Internet space, which broadens the space field, effectively changes the relationship between people and people, people and space, space and space, further improves the efficiency and feedback of information transmission.
\end{abstract}

Keywords: new media art, public space, urban space

\section{INTRODUCTION}

Nowadays, with the rapid development of cities, the flow and communication of information determine the communication cost of development. How to improve the quality of cities by improving the way information communication has become a problem for designers to think about. Combining the concept of space and using digital technology as the carrying mode, the form of new media art is widely used in the exhibition space, public space and urban space, which not only broadens the concept of digital space, but also changes various interactive relations between people and space. With the gradual optimization of digital technology and people's concept of space, there will be more possibilities for the way new media are formed in space in the future.

\section{NEW MEDIA ART AND ITS MAIN CHARACTERISTICS}

New Media Art refers to an emerging discipline that uses digital technology or other media with wider use as creation tools. It includes various forms such as digital art, interactive art, virtual art and bionic art, etc., whose scope is still expanding and constantly integrating with other fields. It is a new art reflecting the existence and cognition of contemporary society and the reconstruction of reality. This subject, which is still in the process of evolution, retains the basic aesthetics of "old media art", also has more excellent artistic forms and performance characteristics, the most important characteristics of which are manifested in technology, interaction, and virtual aspects.

The development of new media art is based on the development of new media technology, and the biggest difference from other art creations is that it exists as a derivative art result of technology. With the development of computer networks, wireless communication networks, satellite and other channel technologies, as well as computer, mobile phone, digital $\mathrm{TV}$ and other terminal technologies, new media art can provide users with information and services. Correspondingly, with the rapid development of new media art, the demand for scientific and technological progress is becoming more and more intense. The popularization and application of information technology such as the Internet of Things, big data, artificial intelligence, and blockchain and so on provide technical reference for new art forms, but also bring more interactive ways of intelligent information.

Traditional art is mostly dominated by artists' selfcenteredness, emphasizing the use of works to convey the author's views and intentions, while new media art no longer continues this one-way information transmission. Interactive design uses information technology to enable the audience to have a strong sense of participation and identity, while designers can also get feedback information for the optimization of 
works and social contributions. Such interaction includes not only the intuitive feeling of physical touch, but also the sensory experience of creating atmosphere and space, thus bringing about the exchange and collision of different cultures, experiences and arts.

New media art uses media and other technical tools to make the original plane or other static threedimensional works of art have vitality, effectively enhance the sense of reality of art and the audience's sense of immersion and participation. Virtual space, virtual things and virtual experience can present the atmosphere that can not be immediately perceived in common sense, as the characteristics of new media art fully shock the viewer's experience.

\section{EXHIBITION SPACE AND NEW MEDIA ART}

The development of art and design always complement each other, the development of science and technology can lead to the innovation of art, and the renewal of art can promote the progress of science and technology. The core of new media art is based on the support of digital technology, and the blending of "virtual" and "reality" brought about by these digital technologies is also a performance feature of new media art. The exhibition space is more suitable for new media art setting than other places. It can use equipment to convey and capture information in the established space. As the entertainment part of urban life is gradually valued by people, the media art design in exhibition space such as art galleries and museums has also entered the field of vision of designers. Shanghai has a "Museum of Art without Maps", which is named "team Lab boundless" and is composed of art groups without boundaries created by the art team Lab. Team Lab Boundless advocates that art goes out of the room, communicate with other works or audiences, break the boundaries between works and audiences in a technical way, and also advocate that they sometimes collide and influence each other. The audience immerses, explores and hesitates in it, and unintentionally participates in the whole huge work and becomes a part of the world. One of the most striking works in the museum, located on Huangpu Riverside, is "The Forest of Breathing Lamps" ("Fig. 1"), which is a display space composed of six mirror walls and dozens of groups of zigzag lamps, the inspiration for this work comes from a premodern Japanese technique called "overlapping colors," that is, the combination of multi-layer silk surface color and back color, forming a changeable color art. Different color atmosphere can be obtained according to the complex ways of overlapping color levels, weaving vertical lines and horizontal lines, and the artist names different series of installation works as color names representing seasons.

When someone stands near the lamp of the work, the lamp closest to the target will emit strong light and sound. The light then travels to the two lamps that are closest in space to the first fixture. The propagated lamp emits the same light and sound, then propagates to the nearest lamp again, and extends continuously. The ingenuity of the work lies in the fact that the light tubes on the transmission path will pass through all the lamps and make each lamp emit light only once, and will return to the original lamp, which makes the space of the work form two unique light paths. The visual effect of principle related to the position of lamps and lanterns is put, designers reasonably choose to anchor point in the space according to the principle of mathematics, the height of the lamp hanging and uneven distribution, the smoothness of the path in the three-dimensional space, and these results all passed the strict test and evaluation of several kinds of schemes, so the rigorous design process can eventually formed this work.

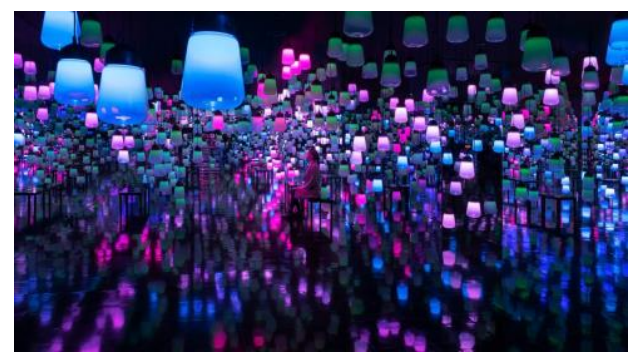

Fig. 1. Echo lamp forest - hydrangea series.

${ }^{\text {a. }}$ Source: Team lab team

The establishment of a new way of experience in the exhibition space, which is different from the simple plane image, is no longer a static beauty created on the premise of fixed space, but also includes the dynamic beauty formed by the interaction between the audience and the device. In this case, the use of the media to convey information creates a new age of space that embraces the changes and dynamics of human behavior. This kind of new media art works make full use of the volume characteristics of space, provide a way for the audience to integrate into the exhibition works, and also provide a new way for designers to display space design.

\section{PUBLIC SPACE AND NEW MEDIA ART}

The new media art in the public space is a kind of way to produce the relationship between art and the public, and it is also a specific place to provide the possibility of communication between them, at the same time, it has the characteristics of both public space and new media art to achieve environmental integration. In recent years, with the upgrading of mobile terminal hardware, the use of augmented reality technology for urban life has become more convenient and frequent. As the requirements of this technology for hardware carriers are no longer strict and can be easily used by the general public, more and more new media 
fields try to integrate this technology into daily life. If art exists everywhere and exists in every corner of public space virtually, it seems to be a preview of the future public space. Artist Ivan Toth Depeña designed a program called "Decoder and Magnifying Glass to Reveal Hidden Treasures in Real Environment" in 2016, which is called "Lapse". The application consists of six interwoven parts: Vision and Collective are two sets of accessible public murals in different locations, Sound is a GPS-based audio soundscape on the MetroMover in downtown Miami ("Fig. 2"), Opus is located in Museum Park in downtown Miami to provide visitors with a virtual prose reading experience, Sculpture a scene for visualizing virtual wind flow by means of a solid public sculpture is provide, Moments is a live exhibition held in the Locust Project space in Miami Design District. With the help of smartphone cameras and GPS positioning systems, Lapse can provide new public space and new media experience for the public, which is limited to viewing in virtual space.

The custom mobile app, which connects six pieces of content, was created by Mr. DePeña with the help of Heavy Projects, a software engineering firm based in Southern California. After downloading the application to mobile terminals, through pioneering augmented reality technology, camera lens and sound output of mobile devices, terminal devices can display virtual visual and auditory experience, respond to the triggers of works and activate each work. Because of the scalability of augmented reality technology, any public with intelligent terminal equipment can participate in this intelligent virtual city experience, and this new media propaganda mode can also let more people experience the charm of science and technology participating in artistic creation.

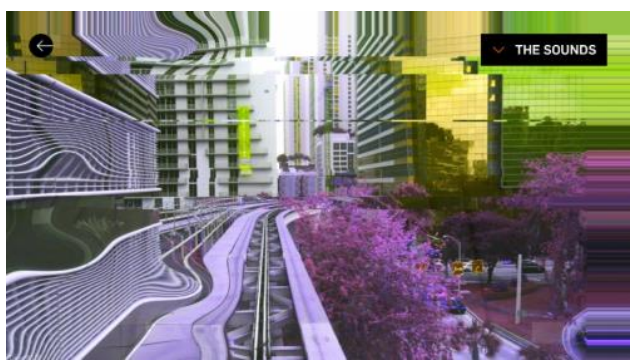

Fig. 2. Audio and video works on downtown Miami Light Rail.

Establishing a new concept of space can expand the space capacity from multiple dimensions, this "digital space" is a digital representation of the real physical space in the network world, which can expand the public space to twice or more, while lifestyles can be transferred to the virtual space for operation. With saving resources, this experimental way of "new media art" can also ensure that space meets the needs of people to explore, and works as aesthetic carriers connecting virtual space and terminal equipment dispel the boundaries between physical reality and the virtual world.

\section{CITY AND NEW MEDIA ART}

The development of real-time data computing and processing technology has made it possible for urban big data to generate visual works. In recent years, a large number of artists have emerged to try to create artistic works in the way of intelligent technology. One of Turkey's media artist and director Refik Anadol is a pioneer in the field of machine intelligence aesthetics. In 2017, he created a place-specific work "The Wind of Boston" ("Fig. 3") in the hall of 100 North Street of Van's Wharf in Boston, the United States, to Boston in and around the invisible state of the Wind into a series of poetic data, these data is presented as an image representation on a digital canvas measuring $13 * 6 \mathrm{~m}$. To get enough data to do the work, Refik Anadol and his team collected a wind data set from Boston's Logan Airport that recorded every 20 seconds for a year, with custom software for a series of intelligent readings, analyses and visualizations of wind speeds, wind directions and patterns, as well as time and temperature.

Created in this way, Wind of Boston consists of four dynamic chapters, each of which data as a source to create four unique forms of visual expression which either abstract or preserve the appearance of the city, to explain the interaction between the environment and the city. The performance of each chapter introduces different aspects of the data set into urban life, and the works have distinct and diverse paintings and perceptual aesthetics, making the intangible beauty of wind emerge as a natural phenomenon that can be simulated.

Each of the four chapters focuses on a unique feature of The Boston Breeze. The first chapter, Hidden Landscapes, highlights the most fundamental readings of wind speeds, creating an immaterial, spatial experience. The second chapter, Memory of Porcelain, breaks through the traditional time limit and lets people reimagine the invisible power of strong winds. Chapter three, The Sea Breeze, explores the paradox of a gentle breeze blowing from sea to land in the depths of winter. The fourth chapter, Gusts of the City, is a highperspective visual work that explores the phenomenon of high-speed winds blowing over a short period of time and the continuous invisible poetic dance between nature and the built environment.

Every day from 8 a.m. to 11 p.m., people can see the work from indoors to outdoors. By defining a new spatial poetics through media art and data visualization, the designer has created an intelligent work of intelligence, memory and culture, turning the wall it sits on into innovative public art. By changing chapters with the passage of time, the work has a direct 
connection with the surrounding environment of the specific city in which it is located, and the public can experience the city that usually ignores but actually exists through the appreciation of the work.

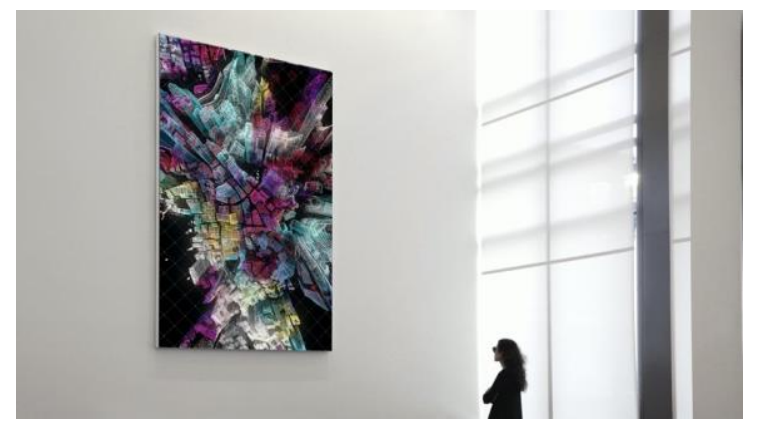

Fig. 3. Photos of "Wind of Boston".

With the improvement of big data information processing ability, more technologies like augmented reality, real-time computing and swarm intelligence have opened up new ideas for new media art to act on urban space. There are a large number of information to be processed in the city every day, which were once ignored as unnecessary contents to be displayed to the public. However, with the development of the city in the information age, the public has the right and need to understand the hidden urban information behind these data. The case mentioned above tries to transform the natural data specific to a city into images, which provides a demonstration for the rapid collection and processing of "smart city" information by new media art works, and also shows how to transmit these results to the public and trigger public thinking about living areas. At the same time, as feedback, the public's intentions for their cities may also be captured and responded to by "smart cities" in the future. The attempt of these cases indicates that there will be more and more excellent intelligent works of new media art in urban living space in the future.

\section{CONCLUSION}

With the continuous progress and development of new media technology, the definition of space is no longer stay in the original geographical space but evolved into a multi-dimensional space based on the Internet. Exhibition space began to break the restrictions of plane and volume, and explore more diverse ways of information transmission between artists and audiences. The boundaries between public places and private places continue to blur, selfsufficiency and business models coexist on the Internet is a new form of contemporary public space. Urban space uses technology and art to show the invisible concept of "city" in the way of new media art and to establish a new way of life in physical space such as commercial places. From the cases mentioned in this paper, we can see that new media art, as a design method, is stationed in the Internet space, broadening the space field, effectively changing the relationship between people, people and space, space and space, and further improving the efficiency and feedback of information transmission. With the promotion and application of media and Internet technology, there may be more space application cases in the future.

\section{References}

[1] Qian Xing. New Media Art: Focus on people, art and life take new media art in public space as an example $[\mathrm{J}]$. The House of Drama,2017(18):102-103.

[2] Zhao Yang. Introduction to new media art in the application of display design $[\mathrm{J}]$. Journal of art and design (theory), 2020, 2 (02): 57-59.

[3] Zhang Xin. Brief Analysis of the Influence of New Media Art on Interaction Design [J]. Peer, 2016(06):202.

[4] Chen Jielu, Wu Lingqin, Li Shan. New Media Art Design Trend Analysis [J]. News Front, 2016(16):145-146.

[5] Wan Yuji. Application of New Media Digital Image Technology in Environmental Art Design Teaching - A Case study of Space Composition and Design [J]. Art and Design (Theory),20,2(01):150-152.

[6] Li Tiemeng. Data Art - a new art form under the current trend of technological thought $[\mathrm{J}]$. Journal of Nanjing University of the Arts (Art and Design), 2019(03):10-13+209. 